\title{
Bone Cement for Ossicular Chain Defects
}

\author{
ESSAM FATEHY, M.D. and WAEL A. ALZAMIL, M.D. \\ The Department of Ear, Nose and Throat, Hearing and Speech Institute, \\ General Organization for Teaching Hospitals and Institutes, Cairo, Egypt
}

\begin{abstract}
Background: The discontinuity of ossicular chain most commonly affects the incudostapedial joint in $80 \%$ of patients an absent incus, or an absent incus and stapes superstructure. This discontinuity leads to a conductive hearing loss of different degrees. The goals of surgery for chronic ear disease are eradication of the disease and reconstruction of a sound transformer mechanism, but reconstructing this defect is a challenging procedure for the surgeon with different treatment options such as total ossicular chain prosthesis, a partial ossicular chain prosthesis, incus interposition, or bone cement utilization. Extrusion of the prosthesis is still a challenge in ossiculoplasty with alloplastic materials, especially in patients with poor eustachian tube function. Bone cement is cost effective, because it is cheaper than other ossiculoplasty materials, and this cost-effectiveness plays an important role in the decision the surgeon has to make, and bone cement has a promising role in this so, there is a rising interest in the otologic surgical field to use bone cement because of its biocompatible profile, its easy application, its suitable cost, and its low extrusion rate. Also, it may have a potentiality of neo-osteogenesis.
\end{abstract}

Aim of Study: To evaluate the post-operative results and usefulness of bone cement application in repairing ossicular chain defects, to discuss surgical technique, and to compare pre-operative and post-operative ABG.

Patients and Methods: This study was carried out in the ORL-HNS Department, Hearing and Speech Institute, from April 2015 to March 2018, on 30 patients with ossicular chain defect. Bridging of ossicular discontinuity was done by glass monomer bone cement in all patients.

Results: In this study, successful hearing restoration could be achieved in $96 \%$ of the patients represented, as $52 \%$ of the patients had hearing improvement ( $\mathrm{dB}$ gain) of more than or equal $10 \mathrm{~dB}$, and $26 \%$ of patients had hearing improvement (dB gain) more than $20 \mathrm{~dB}$, and $18 \%$ of patients had hearing improvement (dB gain) of more than $40 \mathrm{~dB}$, after 12 months.

Correspondence to: Dr. Essam Fatehy, The Department of Ear, Nose and Throat, Hearing and Speech Institute, General Organization for Teaching Hospitals and Institutes, Cairo, Egypt
Conclusion: Incudostapedial joint rebridging ossiculoplasty with ionomeric bone cement is a reliable method for ossicular reconstruction that is cost effective and offers satisfactory hearing results in selected patients.

Key Words: Bone cement-Hearing - Ossicular chain defect.

\section{Introduction}

THE normal ossicular chain functions as an efficient lever system that transfers sound from the tympanic membrane to cochlear fluids [1]. Chronic otitis media commonly affects the middle ear structures with erosion and disconnection of the ossicular chain [2]. The discontinuity most commonly affects the incudostapedial joint (occurring in $~ 80 \%$ of patients with ossicular discontinuity), an absent incus, or an absent incus and stapes superstructure. This discontinuity leads to a conductive hearing loss of different degrees [3]. The goals of surgery for chronic ear disease are eradication of the disease and reconstruction of a sound transformer mechanism [4], but reconstructing this defect is a challenging procedure for the surgeon with different treatment options such as total ossicular chain prosthesis, a partial ossicular chain prosthesis, incus interposition, or bone cement utilization [5] .

Extrusion of the prosthesis is still a challenge in ossiculoplasty with alloplastic materials, especially in patients with poor eustachian tube function [6]. Bone cement is cost effective, because it is cheaper than other ossiculoplasty materials [7], and this cost-effectiveness plays an important role in the decision the surgeon has to make, and bone cement has a promising role in this so, there is a rising interest in the otologic surgical field to use bone cement because of its biocompatible profile, its ease of application in difficult regions, its reasonable cost, and its low extrusion rate. Moreover, it seems to have a potentiality of neo-osteogenesis [6]. 


\section{Patients and Methods}

Patients: The study was carried out in the ORLHNS Department, Hearing and Speech Institute from 2015 to 2018 , on 30 patients with ossicular chain defect. The inclusion criteria consist of patients with chronic ear disease either the turbotympanic type (safe ear) or the attico-antral disease (unsafe ear) who have conductive hearing loss with air-bone gap more than $25 \mathrm{~dB}$, with intraoperative criteria of eroded incus long process, or completely absent incus. Exclusion criteria were absent stapes superstructure, extensive cholesteatoma with intracranial complications or labyrinthine fistula, presence of sensorineural hearing loss, and medically unfit patients for surgery. Pre-operative assessment has been done in the form of detailed patient history inquiring about ear discharge for long periods and hearing impairment with or without tinnitus. Clinical examination in the form of otoscopy, endoscopy, and microscopic examination, in addition to pure tone audiometry and speech audiometry was done. On fulfilling the selection criteria, patients underwent preoperative and postoperative audiologic evaluation measuring airbone gaps at frequencies of $0.5,1,2,3$, and $4 \mathrm{kHz}$ to calculate pure tone averages. All patients completed the follow-up audiometry at 3, 6, and 12 months after surgery.

\section{Material used:}

The glass-ionomer bone cement (GIC) contains a powder composed of glass powder, polycarboxylic acid, as well as a liquid composed of water, or water with other components. This GIC can be used in minor ossicular defects, such as a missing lenticular process, which can be bridged with this ionomer cement. Moreover, this material can be used in the reconstruction of the posterior auditory canal wall and obliteration of the mastoid cavity.

\section{Surgical technique:}

Under general anesthesia, a postauricular approach was used in 28 patients and the permeatal transcanal approach in two patients with localized pathology. In the postauricular approach, an incision was marked $5 \mathrm{~mm}$ posterior to the auricular crease and carried out through the skin and subcutaneous tissue with care not to enter the ear canal. When the temporalis fascia is reached, a graft can be harvested using a Freer elevator and scissors, then a periosteal incision is made in $\mathrm{T}$ or $\mathrm{C}$ fashion, and the periosteum is raised, and the tympanomeatal flap is raised, so the middle ear is accessed. The middle ear workis carried out, identifying the ossicular chain and checking its mobility and continuity to determine the need and type of ossicular reconstruction. According to the state of the ossicular chain, two procedures are available, the I-S or M-S bone cement ossiculoplasty. A few drops of the powder and liquid are poured on a metal plate and mixed. The cooling mixture becomes muddy before it hardens in a couple of minutes. Using a needle, the cement is applied drop by drop to reduce the ossicular gap.

\section{I-S procedure of incus long process reconstruction:}

The components were mixed by hand during the operation. The mucous membrane was removed from the lateral surface of the remnant of the long process with a preparation needle, or a fine diamond burr and a small amount of the cement was placed on the remnant of the incus with a preparation needle Figs. $(1,2)$. This action was repeated several times, if necessary, until the last dose of cement surrounded the stapes head Fig. (3). After setting, fine palpation of the neck of the malleus confirmed the transference of the movements to the stapes, and then tympanic membrane grafting is performed using temporalis fascia Fig. (4). M-S procedure for ossicular chain reconstruction (malleus relocation technique): After dissecting the malleus free from the TM, the tensor tympani were sectioned Fig. (5), and any remnant of the incus was removed. The relocation was done using a strong $90^{\circ}$ hook placed anterior to the neck of the malleus Fig. (6). Subsequent anterior retraction of the malleus was avoided by overstretching the anterior malleal ligament, the position thereby being maintained by the superior ligament of the malleus, which was preserved. In the relocated position, the malleus should lie immediately over the stapes capitulum or suprastructure Fig. (7). Then, the cement is applied to the manubrium of the malleus, preferably at the region of the manubrium between the middle and inferior third, Figs. (8). Then the cement bridge is constructed Fig. (9). Grafting of the tympanic membrane is performed using temporalis fascia graft Fig. (10). 


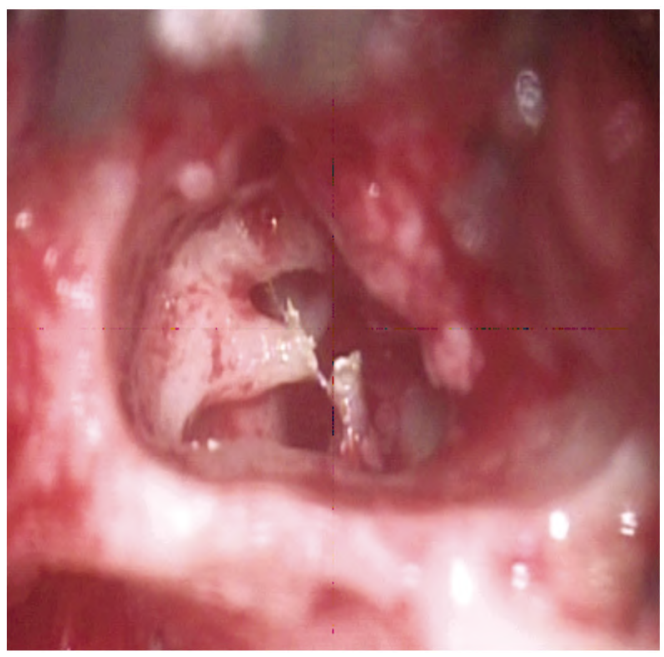

Fig. (1): Absence of lenticular process.

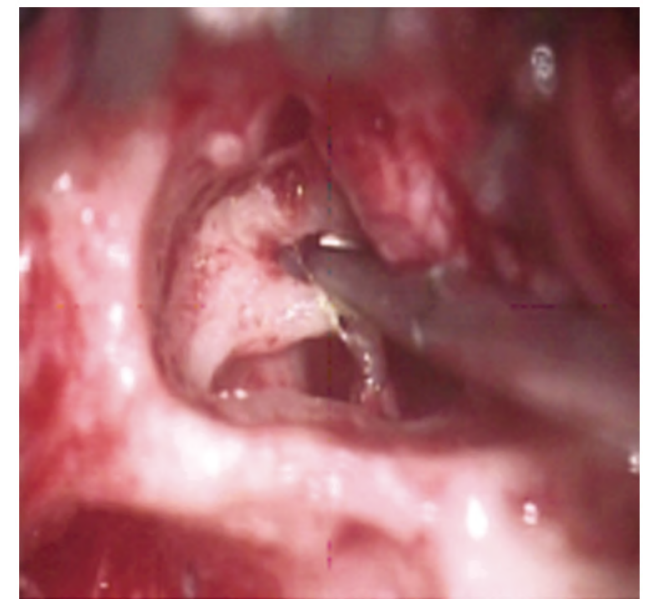

Fig. (3): Ionomer cement bridge between the incus body and the stapes head.

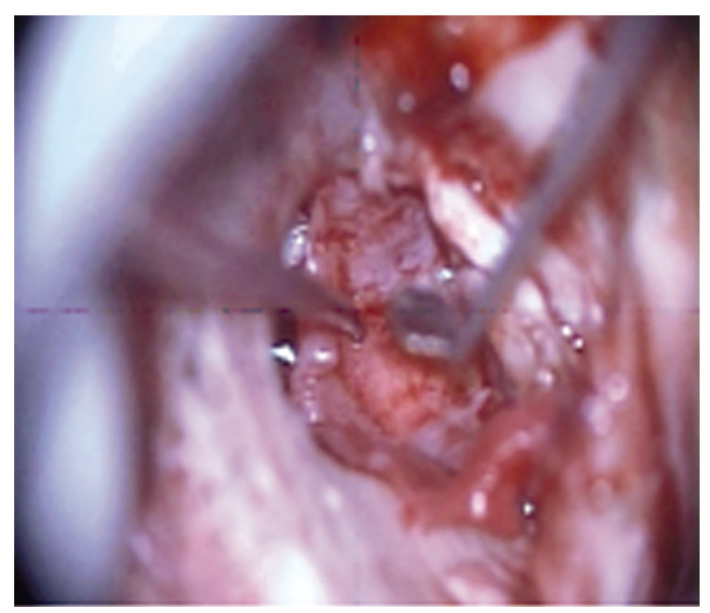

Fig. (5): Absence of incus.

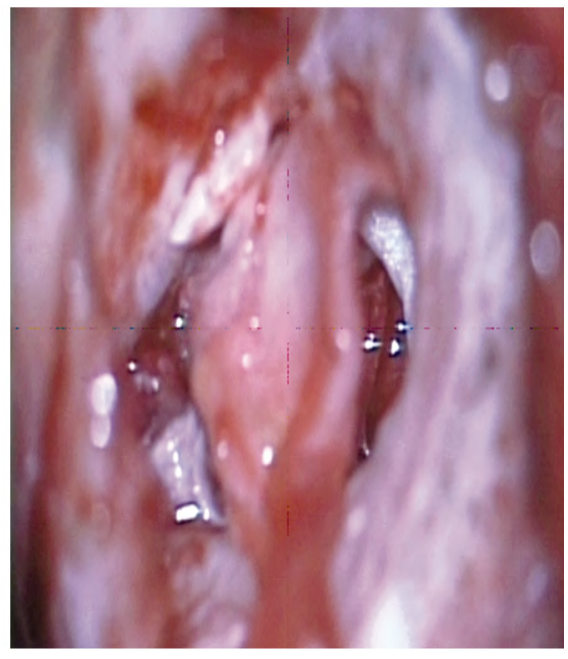

Fig. (2): Cement is placing with a needle.

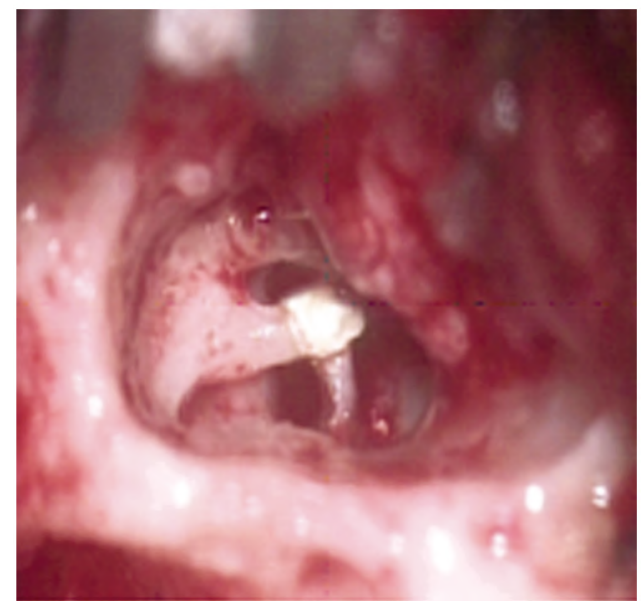

Fig. (4): Tympanic membrane grafting.

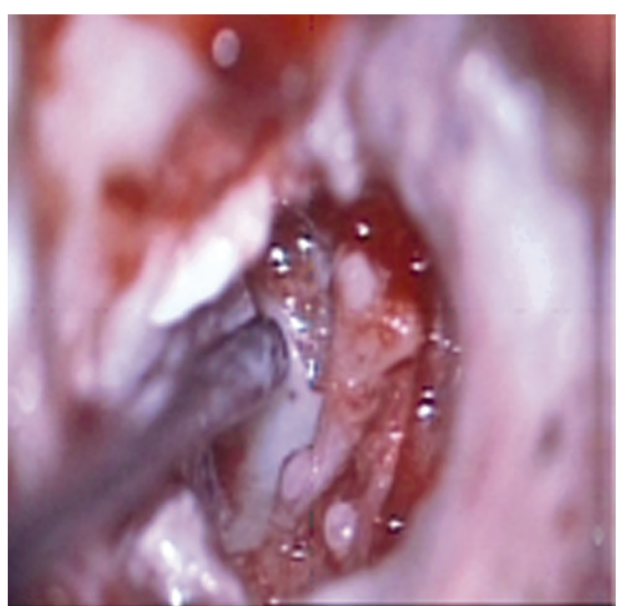

Fig. (6): Malleus relocation. 


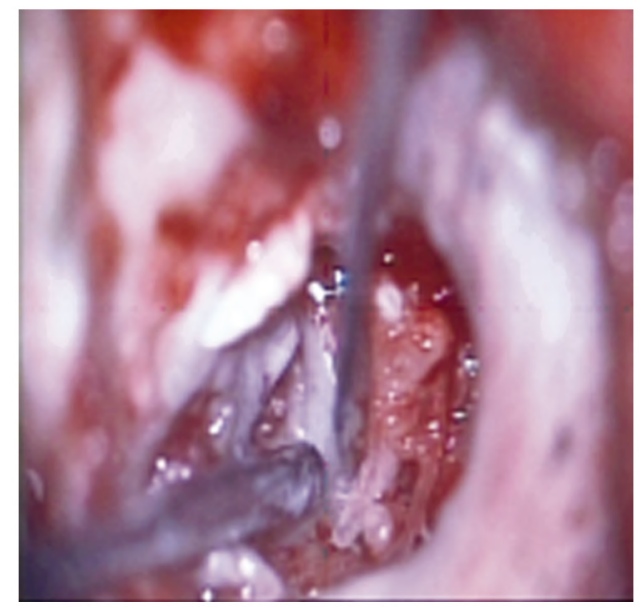

Fig. (7): The relocated malleus above the stapes capitulum.

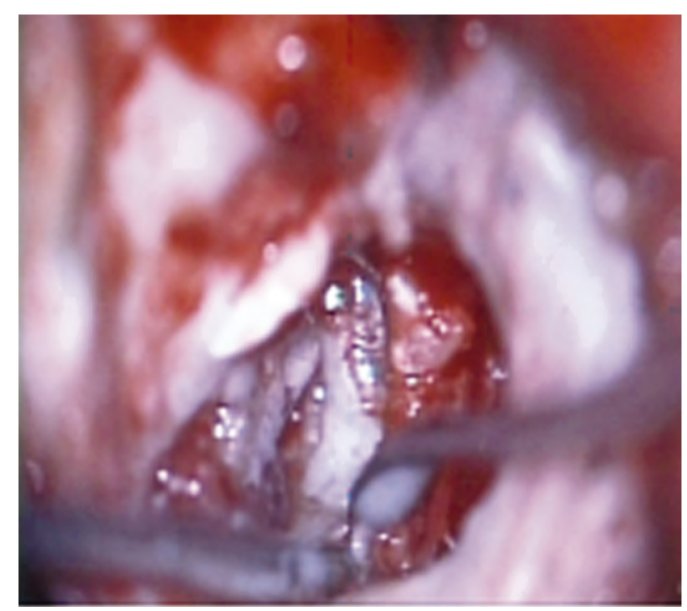

Fig. (9): Lonomer cement bridge between the malleus and the stapes head.

\section{Results}

The study included 23 male and 7 female patients, with overall sex incidence of $77 \%$ males and $23 \%$ females. The age range was from 10 to 64 years, with a mean age of 46 years. The common complaint in all patients was hearing loss. The two procedures of ossicular chain reconstruction done for the patients included in the study are incudostapedial procedure done for 24 patients and the malleuostapedial procedure done for six patients. All patients underwent a pre-operative and postoperative audiologic evaluation for the air-bone gap at frequencies of $0.5,1,2,3$, and $4 \mathrm{kHz}$ to calculate pure tone averages. All patients completed the follow-up audiometry at 3, 6, and 12 months after surgery (Tables 1-3). Comparison according to the average air-bone gap (ABG) pre-operatively and at 3,6 , and 12 months post-operatively is

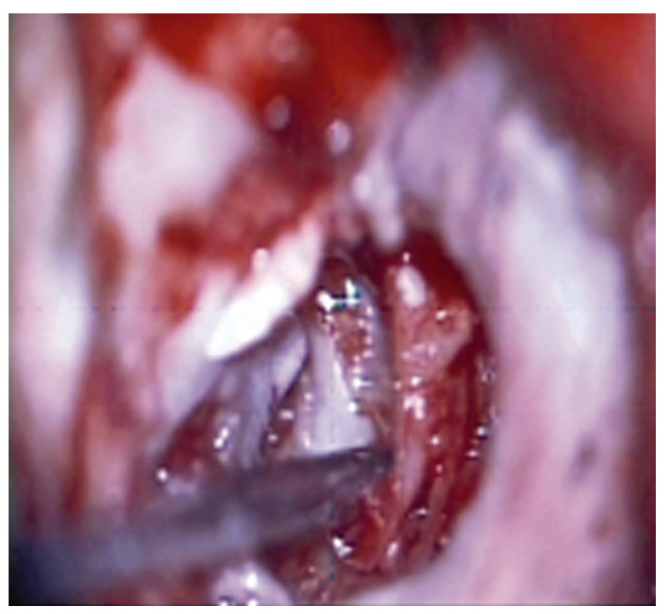

Fig. (8): Cement placing with a needle.

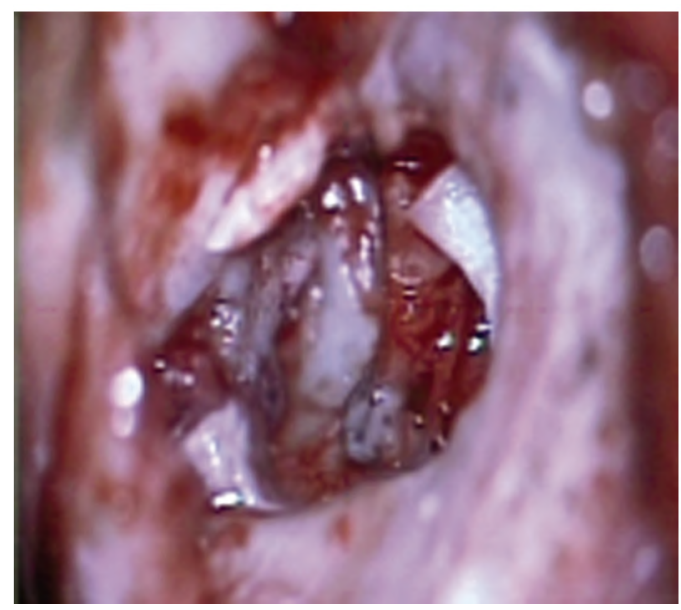

Fig. (10): Tympanic membrane grafting.

shown in (Table 4). Table and diagram show the mean hearing frequencies pre-operatively and at 3,6 , and 12 months post-operatively. Patients who had graft failure and required revision surgery were excluded from the study. There was no serious complication such as facial palsy or sensorineural hearing loss.

Table (1): Stratification of cases according to the average ABG pre-operatively and 12 months postoperatively.

\begin{tabular}{llcc}
\hline & $\begin{array}{c}0-20 \mathrm{~dB} \\
{[\mathrm{n}(\%)]}\end{array}$ & $\begin{array}{c}21-40 \mathrm{~dB} \\
{[\mathrm{n}(\%)]}\end{array}$ & $\begin{array}{c}>40 \mathrm{~dB} \\
{[\mathrm{n}(\%)]}\end{array}$ \\
\hline Pre-operative & 0 & $14(44)$ & $16(56)$ \\
12 months post-operative & $16(52)$ & $14(44)$ & \\
\hline
\end{tabular}

$52 \%$ of patients have hearing improvement with the closure of ABG less than $20 \mathrm{~dB} .44 \%$ of patients have hearing improvement with the closure of $\mathrm{ABG}$ from 21 to $40 \mathrm{~dB}$. $0 \%$ of patients have no hearing improvement with $\mathrm{ABG}$ more than $40 \mathrm{~dB}$. 
Table (2): Stratification of cases according to the average $\mathrm{dB}$ gain 12 months post-operatively.

\begin{tabular}{lcccc} 
dB gain & $\begin{array}{c}0 \mathrm{~dB} \\
{[\mathrm{n}(\%)]}\end{array}$ & $\begin{array}{c}\text { More than } \\
10 \mathrm{~dB} \\
{[\mathrm{n}(\%)]}\end{array}$ & $\begin{array}{c}\text { More than } \\
20 \mathrm{~dB} \\
{[\mathrm{n}(\%)]}\end{array}$ & $\begin{array}{c}\text { More than } \\
40 \mathrm{~dB} \\
{[\mathrm{n}(\%)]}\end{array}$ \\
\hline $\begin{array}{l}12 \text { months } \\
\text { post-operative }\end{array}$ & $16(52)$ & $8(26)$ & $6(18)$ \\
\hline
\end{tabular}

$96 \%$ of patients have hearing improvement with 'dB gain' after 12 months (presented as follow). 52\% of patients have hearing improvement 'dB gain' more than or equal $10 \mathrm{~dB}$. $26 \%$ of patients have hearing improvement 'dB gain' more than $18 \mathrm{~dB}$. $20 \%$ of patients have hearing improvement 'dB gain' more than $40 \mathrm{~dB}$.

Table (3): Stratification of cases according to the mean ABG and SD pre-operatively and post-operatively.

\begin{tabular}{lcccc}
\hline & $\begin{array}{c}\text { Pre- } \\
\text { operative }\end{array}$ & $\begin{array}{l}\text { After 3 } \\
\text { months }\end{array}$ & $\begin{array}{l}\text { After 6 } \\
\text { months }\end{array}$ & $\begin{array}{c}\text { After 12 } \\
\text { months }\end{array}$ \\
\hline Mean ABG & 44.6 & 29.4 & 23.3 & 20.1 \\
SD & 10.7 & 9.8 & 9.7 & 9.2 \\
\hline
\end{tabular}

Table (4): Stratification of cases according to the mean hearing frequencies, mean $\mathrm{ABG}$, and mean speech discrimination pre-operatively and post-operatively.

\begin{tabular}{lcccccc}
\hline & & & & & & Speech \\
& HZ & 1000 & 2000 & 4000 & Average & HZ \\
& & HZ & HZ & ABG & discrimination \\
& & & & & $(\%)$ \\
\hline Pre-operative & 44.7 & 42 & 39.5 & 42.1 & 43.4 & 91.1 \\
After 3 months & 30.2 & 26.8 & 28.5 & 29.4 & 29.2 & 92.6 \\
After 6 months & 26.5 & 24.1 & 22.5 & 26 & 25.3 & 94.2 \\
After 12 months & 23.1 & 20.2 & 20.8 & 21.2 & 21.2 & 94.8 \\
\hline
\end{tabular}

\section{Discussion}

Loss of ossicular continuity is a common problem following chronic middle ear infections, but many different options exist to treat this [8]. This discontinuity results in a conductive hearing loss which may be encountered frequently during otologic surgery. Therefore, ossicular reconstruction is an indispensable part of the hearing restoration [9], to minimize $A B G$ and maximizing system stability as a major goal to reach this aim [10]. Zollner [11] first described the reconstruction of the ossicular chain in 1955 . Since then, several other attempts have been made, and several material, biologic and synthetic, have been proposed for ossiculoplasty [12]. This reconstruction of the ossicular defects can be accomplished by various methods $[9,10]$. There are three main types of ossicular chain reconstruction: Autograft, homograft, and allograft prostheses [8]. Autograft reconstruction has a low extrusion rate, no risk of transmitting disease (contrary to homograft), and has proven biocompatibility, and additionally, it is a cheap option compared with allograft-prosthesis. Disadvantages include displacement. For some ossicular chain problems, bone cement can be used to restore these defects as a cost-effective option $[6,10,13]$ The challenge during ossiculoplasty was mainly, how to achieve a stable, reliable connection between the tympanic membrane and mobile stapes footplate with a material that will provide the best long-term hearing results, without complications (Celik et al., 2001), in addition to the low cost of this material to achieve cost-effectiveness, which plays an important role in the decision the surgeon has to make [8]. Hoffmann et al., [14] demonstrated already that bone cement could bind adequately to the ossicular bone with new bone growth and no inflammation occurring at the site of application, making a suitable solution as an agent to fix the sculpted autograft to the remaining ossicular chain. Ossiculoplasty with bone cement is a cheap and simple procedure. It is known to be safe and not affecting the inner ear functions, and there is also a proof of biocompatibility and osseointegration [15]. Bone cement is cost-effective because it is cheaper than other ossiculoplasty materials [7]. In this study, we present here GIC to elevate postoperative hearing gain in the case of ossicular disconnection. Since the first bone cement used for otologic surgery, some studies especially reported its usage for defective incus long processes as an incudostapedial bridge, providing good hearing results. These results are plausible, because incudostapedial rebridging with bone cement maintains the normal anatomy $[\mathbf{6 , 8 , 1 0 , 1 3 ]}$. GIC has been applied in otology since 1989 [16]. It was employed in stapes surgery for additional fixation of the prosthetic loop and in ossiculoplasty for bridging discontinuities [17]. GIC brings the possibility of ossicular repair because of the following characteristics that make it suitable for use in the middle ear. It adheres to bone and metal, it is moldable before it sets, after setting it can be drilled, there is minimal exothermic reaction, there is no shrinkage after setting, it has a quick setting time, and it is stable in a wet environment after set [18].

Disintegration in the incudostapedial joint represents the most common ossicular separation encountered in otology [19]. So, incudostapedial rebridging ossiculoplasty is the most common indication of bone cement ossiculoplasty. Bone cement also can be used in different situations such as between malleus and stapes in the absence of incus [13]. Therefore, two procedures are available; the I-S or M-S bone cement ossiculoplasty is performed. In the I-S procedure, the cement is applied drop by drop to reduce the ossicular gap between the incus and stapes head in the I-S procedure (Yildirim et al., 2005) [20]. In the M-S procedure, the malleus was positioned directly over the stapes. In practice, this anatomic configuration is very 
rarely seen but can be easily achieved with the malleus relocation technique as demonstrated in this study. Cement is applied to the manubrium of the malleus, preferably at the region of the manubrium between the middle and inferior thirds. Then the cement bridge is constructed until the head of the stapes is reached. Grafting of the tympanic membrane is performed using temporalis fascia before or after the I-S procedure. However, grafting should precede the M-S procedure (Vlaming and Feenstra, 1986) [21]. The age of the patients in our study ranged from 10 to 64 years, with a mean of 46 years. The study included 23 male and 7 female patients, with sex incidence of $77 \%$ males and $23 \%$ females. The follow-up period was 12 months. Overall, $60 \%$ of our study population achieved a good hearing result of an ABG up to $20 \mathrm{~dB}$. In this study, successful hearing restoration could be achieved in $96 \%$ of the patients, represented as $52 \%$ of patients had hearing improvement $(\mathrm{dB}$ gain) of more than or equal $10 \mathrm{~dB}$, and $26 \%$ of patients had hearing improvement ( $\mathrm{dB}$ gain) of more than $20 \mathrm{~dB}$, and $18 \%$ of patients had hearing improvement (dB gain) of more than $40 \mathrm{~dB}$, after 12 months. Hearing results of different aural pathologies (chronic otitis media and conductive hearing loss) and surgeries (tympanotomy and tympanoplasty with or without mastoidectomy) reveal that there is a highly significant hearing improvement after 12 months.

\section{Conclusion:}

Ossiculoplasty is the reconstruction of the middle ear ossicular chain that has been disrupted or destroyed using some interpositioned devices which help in regaining the original mechanics of the ossicular chain to transfer the sound energy to the inner ear. Bone cement is a good and cheap option for some ossicular chain problems to achieve successful hearing improvement. It can firmly bind ossicular bone and allows new bone to grow at the site of application. Bone cement has many desirable properties that may make it useful as an adjunct to ossicular chain reconstruction. Potential applications for this bone cement include not only anchoring a native ossicle or prosthesis but also repairing a fractured ossicle or reconstructing a shortened ossicle. The ability to firmly bind ossicular bone and allow new bone growth at the site of application with little or no inflammatory reaction makes cement an attractive candidate for continued study [14]. Repair of defects of the ossicular chain with GIC is an easy, efficient, quick, and inexpensive method, which ought to be kept in mind for future middle ear surgery. No complications in the middle ear were related to GIC.

\section{References}

1- GOODHILL V.: The fixed malleus syndrome. Trans. Am. Acad. Ophthalmol. Otolaryngol., 70: 370-80, 1966.

2- O'REILLY R.C., CASS S.P., HIRSCH B.E., KAMERER D.B., BERNAT R.A. and POZNANOVIC S.P.: Ossiculoplasty using incus interposition: Hearing results and analysis of the middle ear risk index. Otol. Neurotol., 26: 853-8, 2005.

3- SEMAAN M.T. and MEGERIAN C.A.: The pathophysiology of cholesteatoma. Otolaryngol. Clin. North Am., 39: 1143-59, 2006.

4- BAYAZIT Y., GOKSU N. and BEDER L.: Functional results of plastipore ossiculoplasty prostheses for middle ear ossicular chain reconstruction. Laryngoscope, 109: 709-11, 1999.

5- CHOLE R.A. and SKARADA D.J.: Middle ear reconstruction techniques Otolaryngol. Clin. North Am., 32: 489-503, 1999.

6- BABU S. and SEIDMAN M.D.: Ossicular reconstruction using bone cement. Otol. Neurotol., 25: 98-101, 2004.

7- FEGHALI J.G., BARRS D.M., BEATTY C.W., CHEN D.A., GREEN Jr. J.D., KRUEGER W.W., et al.: Bone cement reconstruction of the ossicular chain: A preliminary report. Laryngoscope, 108: 829-36, 1998.

8- CELIK O., YALÇıN S. and ÖZTÜRK A.: Hearing parameters in noise exposed industrial workers. Auris Nasus Larynx, 25: 369-75, 1998.

9- ZHANG L.C., ZHANG T.Y., DAI P.D. and LUO J.F.: Titanium versus nontitanium prostheses in ossiculoplasty: A meta-analysis. Acta Otolaryngol., 131: 708-15, 2011.

10- OZER E., BAYAZIT Y.A., KANLIKAMA M., MUMBUC S. and OZEN Z.: Incudostapedial rebridging ossiculoplasty with bone cement. Otol. Neurotol., 23: 643-6, 2002.

11- ZÖLLNER F.: The principles of plastic surgery of the sound-conducting apparatus. J. Laryngol. Otol., 69: 637$52,1955$.

12- QUARANTA N., FERNANDEZ S., FEIJOO V., et al.: Closed tympanoplasty in cholesteatoma surgery: Longterm (10 years) hearing results using cartilage ossiculoplasty. Eur. Arch. Otorhinolaryngol., 258: 20-4, 2001.

13- BAYAZIT Y.A., OZER E., KANLIKAMA M., et al.: Bone cement ossiculoplasty: Incus to stapes versus malleus to stapes cement bridge. Otol. Neurotol., 26: 364-7, 2005.

14- HOFFMANN K.K., KUHN J.J. and STRASNICK B.: Bone cements as adjuvant techniques for ossicular chain reconstruction. Otol. Neurotol., 24: 24-8, 2003.

15- BAHMAD F. and MERCHANT S.N.: Histopathology of ossicular grafts and implants in chronic otitis media. Ann. Otol. Rhinol. Laryngol., 116: 181-91, 2007.

16- HEHL K., SCHUMANN K., BECK C. and SCHOTTLE W.: Use of glass ionomer cement in surgery of the incusstapedial joint. An initial report of experiences. Laryngorhinootologie, 68: 490-2, 1989.

17- GEYER G. and HELMS J.: Ionomer cement prostheses in reconstructive middle ear surgery. HNO, 5: 442-7, 1997. 
18-DOUGLAS C.A. and MOISÉS A.A.: Technical refinements and precautions during ionomeric cement reconstruction of incus erosion during revision stapedectomy. Laryngoscope, 113: 848-52, 2003.

19- GOEBEL A.J. and JACOB A.: Use of Mimix hydroxyapatite bone cement for difficult ossicular reconstruction. Otolaryngol Head Neck Surg., 132: 727-34, 2005.
20- YILDIRIM A., YASAR M., BEBEK A.I., CANBAY E. and KUNT T.: Nasal septal suture technique versus nasal packing after septoplasty. Am. J. Rhinol. Allergy, 19: 599602, 2005.

21- VLAMING M.S. and FEENSTRA L.: Studies on the mechanics of the reconstructed human middle ear. Clin. Otolaryngol. Allied Sci., 11: 411-22, 1986.

\section{إستخدام آسمنت العظم فى علاج

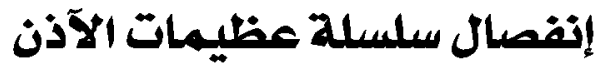

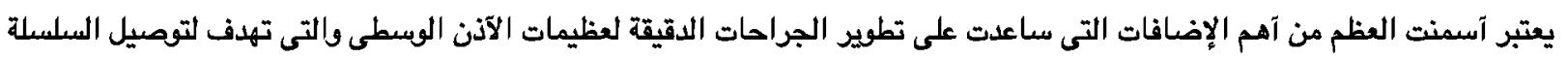

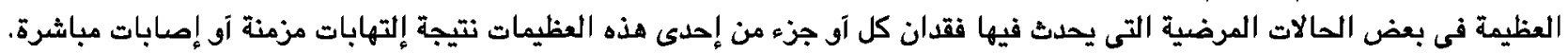

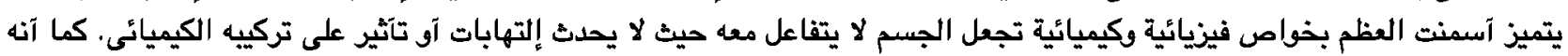

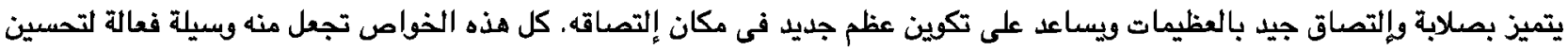
السمع فى حالات الضعف السمعى التوصيلى الناتج عن إنفصال عظيمات الآنن. 